$9-2020$

Tuberculosis of the flexor carpi radialis muscle - A case report

Shah Fahad

Naveed Baloch

Nasir Ud Din

Follow this and additional works at: https://ecommons.aku.edu/pakistan_fhs_mc_surg_orthop

Part of the Bacterial Infections and Mycoses Commons, Pathological Conditions, Signs and Symptoms Commons, and the Pulmonology Commons 


\title{
Tuberculosis of the flexor carpi radialis muscle - a case report
}

\author{
Shah Fahad', Naveed Baloch², Nasir Ud Din ${ }^{3}$
}

\begin{abstract}
Tuberculosis involving almost any organ of the body is a major problem in developing countries. The most commonly affected site in tuberculosis is the lung. Of the extra pulmonary tuberculosis, musculoskeletal involvement accounts for $1-5 \%$ of the cases. Muscular involvement in tuberculosis is a rare manifestation of extra pulmonary tuberculosis. Unusual presentations often go undetected and are associated with a diagnostic and therapeutic delay. This case report presents tuberculosis of the flexor carpi radialis.
\end{abstract}

Keywords: Tuberculosis, flexor carpi radialis muscle, antituberculous drugs

\section{DOI: https://doi.org/10.5455/JPMA.40799}

\section{Introduction}

Tuberculosis involving almost any organ of the body is a major problem in developing countries. The most commonly affected site in tuberculosis is the lung. Of extra pulmonary tuberculosis, musculoskeletal involvement accounts for $1-5 \%$ of the cases. Muscular involvement in tuberculosis is a rare manifestation of extra pulmonary tuberculosis.1 This case report presents tuberculosis of the flexor carpi radialis.

\section{Case report}

In August 2016 a 45-year old housewife presented in the outpatient Orthopaedic clinic at the Aga Khan University Hospital, Karachi with a history of swelling on the anterior aspect of her right forearm for the last three months and was experiencing pain for the last one month. This swelling was initially painless, but gradually over the last one month became painful. The patient's medical history did not indicate any trauma, fever, night sweats, weight loss or tuberculosis contact. Neither did the patient benefit from the prescribed short antibiotic and analgesic courses. A physical examination indicated the patient to be vitally stable and in good health.

A $6.3 \mathrm{~cm}$ doughy swelling with an erythematous border was present on the radial aspect of the anterior forearm. Movements of the wrist were painful and restricted, however the movements of fingers were normal and

1,2Department of Surgery, Aga Khan University, Karachi, Pakistan; 3Department of Pathology, Aga Khan University, Karachi, Pakistan Correspondence: Shah Fahad. e-mail: shah.fahad@aku.edu

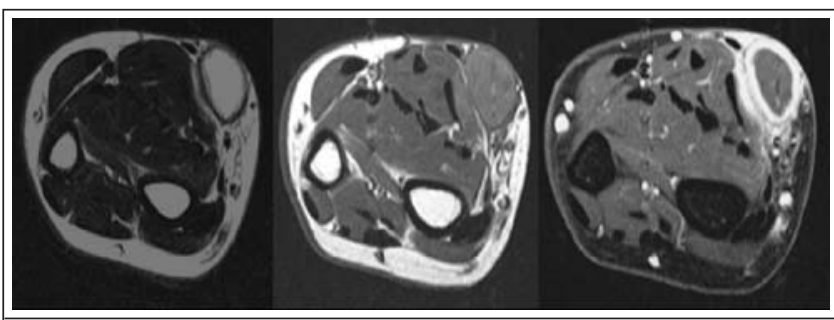

Figure-1: MRl forearm T1, T2 and T1 + C sagittal images which show complete tear and retracted belly of flexor carpi radialis muscle and surrounding inflammatory changes.

comfortable. Sensations in the hand and forearm were intact and pulse at the wrist was normal. Finkelstein test and Tinel's sign were negative.

The total leucocyte count was $6.4 \times 10^{9} / \mathrm{L}$ with $26.6 \%$ lymphocytes. The Erythrocyte sedimentation rate was $04 \mathrm{~mm} / 1$ st hour and the $C$ Reactive Protein was 0.12 . Plain radiographs of the forearm were normal. The magnetic resonance imaging of the right forearm with contrast reported a complete rupture of the tendon of flexor carpi radialis with retraction of the muscular belly and collection in the distal part of retracted belly of flexor carpi radialis (Figure 1).

Treatment comprised of surgical intervention. An incision was made over the swelling and $10 \mathrm{ml}$ of pus was drained. Synovial sheath of the flexor carpi radialis muscle was thick with a gritty sensation on cutting; muscle belly of flexor carpi radialis was necrosed. The clinically involved muscle

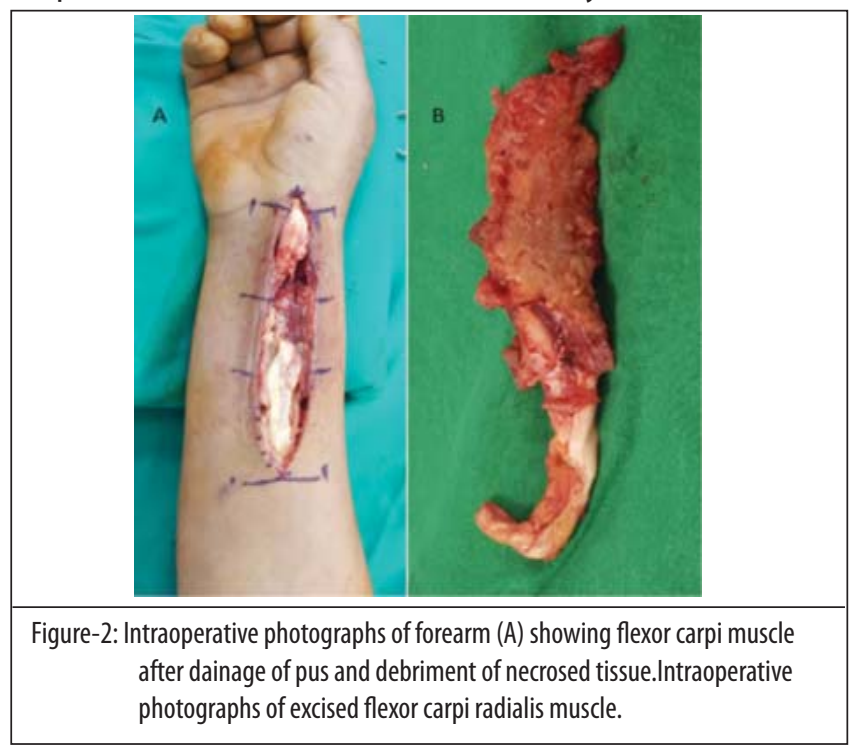




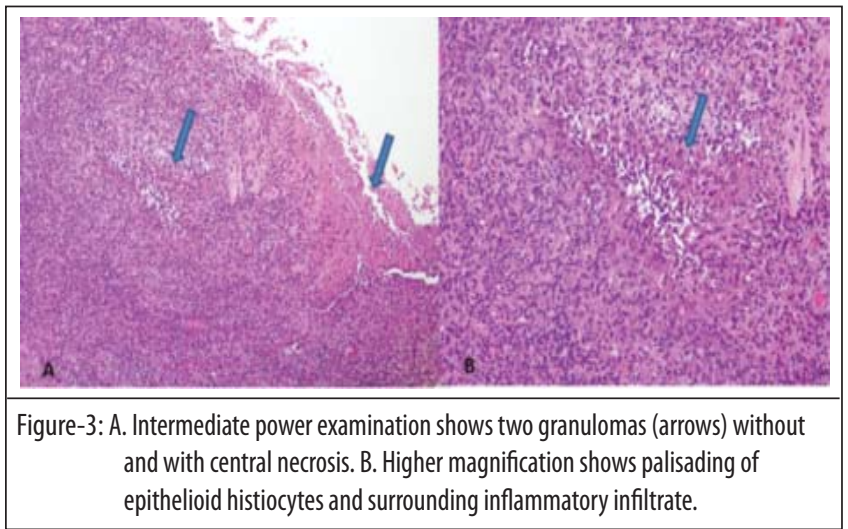

and synovial tissue was excised (Figure 2). The pus was sent for a culture, sensitivity and a genetic evaluation, whereas the muscle belly and synovial sheath were sent for histopathology. Wound was closed primarily. Culture of the pus did not report growth of any organism and the Gene Expert was negative for mycobacterium tuberculosis. Histopathology showed scattered granulomata composed of palisading of epithelioid histiocytes and scattered Langerhan cells with multinucleated giant cells in the synovial sheath and muscle belly. These granulomata were surrounded by lymphoplasmacytic infiltrate. Focal central necrosis was noted (Figure $3 \mathrm{~A}, \mathrm{~B}$ ).

Infectious disease specialist consultation was provided and empirical antituberculous drugs consisting of rifampicin, isoniazid, pyrazinamide, and ethambutol were prescribed for the first two months followed by a combination of rifampicin and isoniazid for the next four months. The wound healed adequately and the patient resumed her daily life activities.

\section{Discussion}

Tuberculous involvement of musculoskeletal tissue is an uncommon form of extrapulmonary tuberculosis accounting for 1-5\% of extrapulmonary tuberculosis cases. Bone is a common site for tuberculous involvement, whereas Tuberculosis Tenosynovitis is a rare manifestation of musculoskeletal tuberculosis. ${ }^{1}$ In rare cases the synovial sheath of the palm is also involved along with the wrist ${ }^{4}$. In this case the flexor carpi radialis was involved but without any extension to the synovial sheath of the hand.

Skeletal muscles are usually spared by tuberculosis because it is a poor host for mycobacterium tuberculosis 2 but they are usually secondarily infected by the underlying bone or synovial tissue of a nearby joint, direct inoculation or haematogenous spread. ${ }^{3}$

The most important step in the diagnosis of tuberculous involvement of the skeletal muscle is a high clinical suspicion, especially in an endemic area. Due to an insidious onset and nonspecific symptoms, the diagnosis of Tuberculous Tenosynovitis is delayed. In most cases the typical presentation of tuberculosis is not seen and constitutional symptoms like weight loss, night sweat, low grade fever are absent.3,4 In this case the typical presentation was absent and the only positive finding in her history is that the patient belongs to an endemic area.

A MRI is required for a detailed assessment of the muscle, tendon and its sheath. At the early stage of the disease the findings from MRI show non-specific synovitis presenting as a serous exudate. However as the disease progresses, the synovium appears thick. The tendon involved becomes thin and may be disrupted. The fungoid stage is characterised by extension of the infection beyond the synovial tissue with obliteration of synovial tissue and formation of a cold abscess. Soft tissue swelling with or without calcification is the common manifestation of tenosynovitis on plain radiographs 5

Tuberculosis of the forearm muscles is a rare entity. To our knowledge there are only a few case reports of tuberculous involvement of the forearm muscles such as a series of three cases reported by Kumar Sen et al. ${ }^{3}$ in one of his case brachioradialis was involved, while two others had the involvement of the forearm muscle with raised inflammatory markers and a bone involvement in the second case. In this case the only muscle that was involved was flexor carpi radialis without affecting any other part of body and the inflammatory markers were normal. To our knowledge there are no other study reports of tuberculous involvement of the flexor carpi radialis muscle as the primary focus of infection.

Three stages are defined by Kanavel in tendenous tuberculosis $^{6}$. Stage I, the hygromatous form is characterised by the presence of a serous exudation with thickening of tendon sheaths. Stage II, the serofibrinous form is characterised by granuloma tissues made of "seed of rice" aspects, and in stage III, the fungoid stage is characterised by the presence of massive necrosis, obliteration of the tendon and sheath, with formation of sinus tracts and a cold abscess. Our case was characterised by the necrosis in the tendon thickness, corresponding to the stage III as reported by Kanavel. ${ }^{6}$

The treatment of the musculoskeletal tuberculous tenosynovitis is typically medical, based on standard protocols, comprising of 6-9 months of rifampicin containing chemotherapy. ${ }^{7}$ In this case the treatment period was 6 months with positive results and without any recurrence within 2 years. The only primary surgical treatment considered was a minor one for a biopsy and for drainage of the necrotic extended lesions, or in the event 
of nerve compression and in case the medical treatment failed. 4,8

\section{Conclusion}

Tuberculosis of skeletal muscle is a rare medical occurrence. It evolves insidiously with a poor clinical presentation and is difficult to diagnose. A high index of suspicion is required to diagnose such a case. MRI is a useful method to guide the diagnosis but a definitive diagnosis is made by histology. Treatment is based on antituberculous chemotherapy and may require surgery in complicated cases which include necrosis and abscess formation.

Patient consent and ethical approval: Written informed consent was obtained from the patient for publication of this case report and any accompanying images.

Disclaimer: None declared.

Conflicts of interest: None declared.

Funding Sources: None declared.

\section{References}

1. Fnini S, Ouarab M, Rafai M, Cohen D, Largab A, Trafeh M. An uncommon occupational accident: tuberculous tenosynovitis of the extensor tendons of the hand. Chirurgie de la Main. 1999; 18:309-12.

2. Kim JY, Park YH, Choi KH, Park SH, Lee HY. MRI of Tuberculous Pyomyositis. J Comput. 1999; 23:454-7.

3. Sen RK, Tripathy SK, Dhatt S, Saini R, Aggarwal S, Agarwal A. Primary tuberculous pyomyositis of forearm muscles. Indian J Tuberc. 2010; 57:34-40.

4. Benchakroun $M, E I A B$, Zaddoug $O$, Kharmaz M, El MY, Ouadghiri $M$, et al. Tuberculosis of the wrist. Symptoms and outcome in eleven cases. Rev Chir Orthop Reparatrice Appar Mot. 2004; 90:337-45.

5. Sanders CJ, Schucany WG. Tuberculous tenosynovitis. Bayl Univ Med Cent. 2008; 21:71-2.

6. Kanavel AB. Tuberculous tenosynovitis of the hand: a report of fourteen cases of tuberculous tenosynovitis. Surg Gynecol Obstet. 1923; 37:635-47.

7. Beam E, Vasoo S, Simner PJ, Rizzo M, Mason EL, Walker RC, et al. Mycobacterium arupense flexor tenosynovitis: case report and review of antimicrobial susceptibility profiles for 40 clinical isolates. J Clin Microbiol. 2014; 52:2706-8.

8. Sbai MA, Benzarti S, Boussen M, Maalla R. Tuberculous flexor tenosynovitis of the hand. Int J Mycobacteriol. 2015; 4:347-9. 